\title{
EFFECT OF NOBILETIN ON EXPERIMENTAL MODEL OF EPILEPSY
} Background: The effects of nobiletin, a plant-derived flavonoid was examined against pentylenetetrazole (PTZ)induced seizures. The study also aimed to assess whether nobiletin potentiated the effects of antiepileptic drug clonazepam (CZP). Methods: PTZ (92 mg/kg, subcutaneous) was used to induce seizures in mice. Treatment groups ( $n=18 /$ group) received nobiletin $(12.5,25$, or $50 \mathrm{mg} / \mathrm{kg}$ ) via oral gavage for 6 consecutive days and 45 min prior to PTZ injection. CZP $(0.015-2.0 \mathrm{mg} / \mathrm{kg})$ was administered $15 \mathrm{~min}$ prior to PTZ. Skeletal muscle strength was assessed by measuring grip strength and Chimney test was performed to study the motor performance in animals. TUNEL assay was done to study neuro-apoptosis. RT-PCR and Western blot analysis were performed for assessment of mRNA and protein expressions. Results: Nobiletin and CZP improved muscle strength and motor coordination and reduced seizure severity significantly. The administration of nobiletin and CZP, individually or in combination, downregulated seizure-induced increases in apoptotic cell count and apoptotic protein expression, modulated the expression of gamma-aminobutyric acid (GABA)A and glutamate decarboxylase 65 and restored the glutamate/GABA balance. Nobiletin and CZP administration significantly upregulated phosphoinositide 3-kinase/protein kinase B (PI3K/Akt) signaling. Conclusion: Nobiletin exerted protective effect against seizures by regulating signaling pathways associated with epileptogenesis and potentiated the effects of CZP.

Keywords anticonvulsant • clonazepam • epilepsy $•$ gamma-aminobutyric acid $•$ nobiletin

\section{Introduction}

Epilepsy is a prevalent neurological disorder characterized by recurrent unprovoked seizures [1]. The seizures are resistant to currently available antiepileptic drugs (AEDs) in around $30 \%$ of patients [2]. There is a demand for novel therapeutic agents and more effective treatment approaches, such as concurrent administration of two or more AEDs [3,4]. There is accumulating evidence of conventional AED side effects, including cognitive dysfunction, recurrent seizures, and psychiatric problems [5]. Therefore, the development of drugs with great antiepileptic potential and negligible or no side effects is crucial for the treatment of epilepsy.

A better understanding of the pathology underlying seizure initiation and progression and associated factors would be useful for the identification of molecular targets for novel AEDs. The experimental induction of seizures with pentylenetetrazole (PTZ), a chloride $\left(\mathrm{Cl}^{-}\right)$ channel blocker on gamma-aminobutyric acid $(G A B A)_{A}$ receptors, is a widely used experimental model for epilepsy $[6,7]$. GABA, the primary inhibitory neurotransmitter, and its receptors mediate inhibitory neurotransmission and prevent neuronal overexcitation [8]. Glutamic acid decarboxylase 65 (GAD65) catalyzes the conversion of glutamate to GABA through decarboxylation [9]. The expression of GAD and GABA receptors is closely associated with epileptic conditions [10-13]. Brain-derived neurotrophic factor (BDNF), upon binding to the tropomyosin receptor kinase $B$ (TrkB) receptor, activates several downstream protein kinases that, in turn, activate target proteins that stimulate the presynaptic release of mediators or modify postsynaptic receptors, including $\mathrm{GABA}_{\mathrm{A}}[14,15]$. Furthermore, BDNF regulates phosphatidylinositol 3-kinase (PI3K)/ Akt signaling [16], which is crucial for synaptic plasticity, and plays a key role in learning and memory formation $[17,18]$.

Clonazepam (CZP), a widely used AED $[19,20]$, affects GABA function and exerts anticonvulsant effects. CZP binding to the benzodiazepine site of $G_{A B A}$ receptors increases $\mathrm{Cl}^{-}$influx in neurons, which inhibits synaptic transmission [21]. However, the usefulness of CZP as a standard treatment for
Baowang Yang ${ }^{1}$

Jing Wang ${ }^{1}$

Ni Zhang ${ }^{2 *}$

'Department of Pediatric Intensive Care Unit, Lanzhou University Second Hospital, Lanzhou, Gansu, 730030, China.

2Department of Pediatrics, Lanzhou University Second Hospital, Lanzhou, Gansu, 730030, China.

Received 02 July 2018

accepted 30 October 2018 epilepsy is limited by its potential side effects, including sedation and resistance related to long-term drug use [19]. Recent research has focused on the antiepileptic effects of plant-derived compounds [22,23]. Nobiletin, a flavonoid found in citrus peels, reportedly possesses several bioactive properties, including anticancer [24,25], anti-inflammatory [26], and neuroprotective activities [27,28]. We investigated the antiepileptic effect of nobiletin administered alone and in combination with CZP following PTZ-induced seizures in mice.

\section{Materials and Methods}

\section{Reagents and chemicals}

PTZ (P6500) (Sigma-Aldrich, St. Louis, MO, USA) was dissolved in freshly prepared saline solution prior to administration. Antibodies against Akt, p-Akt, GSK-3 $\beta$, mTORc1, p-mTORc1, mTORc2, p-mTORc2, cleaved caspase-3, BDNF, TrkB and nGAD65 were purchased from Cell Signaling Technology (Danvers, MA, USA). $\mathrm{GABA}_{\mathrm{A}^{\prime}}$ p-GSK-3 $\beta$, Phosphatase and tensin homolog (PTEN), Bax, BCl-2, Bad, Bcl-xL and $\beta$-actin were purchased from Santa Cruz 
Biotechnology (Santa Cruz, CA, USA) and were used for expression analysis. Nobiletin and the remaining chemicals were obtained from Sigma-Aldrich.

\section{Animals}

C57BL/6 mice (23 \pm 2 g; 5-6 weeks old) were housed under standard conditions $\left(23 \pm 1{ }^{\circ} \mathrm{C}\right.$; 55-60\% humidity; $12 \mathrm{~h}$ light/dark cycle). The mice had ad libitum access to standard mouse food pellets and water and were acclimatized for $5 \mathrm{~d}$ to the environmental conditions prior to the experiments. The study and experimental design were approved by the Animal Studies Ethical Committee of Liaocheng People's Hospital, and the protocols used and handling of animals were in strict accordance with international guidelines [29].

\section{Seizure induction}

The mice were randomly divided into 9 groups ( $n=18$ /group). Individual groups received nobiletin $(12.5,25$, or $50 \mathrm{mg} / \mathrm{kg})$ administered via oral gavage for 6 consecutive days and $45 \mathrm{~min}$ prior to PTZ injection. The doses were based on previous studies conducted in our laboratory (data not shown). PTZ was dissolved in freshly prepared saline solution at a volume of $0.005 \mathrm{~mL} / \mathrm{g}$ of body weight and administered subcutaneously (s.c.) into a loose fold of skin in the midline of the neck.

CZP (freshly prepared in a $1 \%$ solution of Tween 80 in distilled water for each use) was administered intraperitoneally (i.p.) as a single injection in a volume of $5 \mathrm{~mL} / \mathrm{kg} 15$ min prior to PTZ administration, behavioral tests, and tissue analysis for protein expression [6, 30-32]. CZP doses were between 0.015 and $2.0 \mathrm{mg} / \mathrm{kg}$

[31]. Control animals did not receive nobiletin or PTZ but were administered an equal volume of saline solution. Mice treated with PTZ but not nobiletin served as epileptic controls. PTZ (92 $\mathrm{mg} / \mathrm{kg}$, s.c.) was administered to induce clonic seizures. This dose reportedly produces clonic seizures in $97 \%$ of mice [31]. Different groups of mice were treated with nobiletin $(12.5,25$ or $50 \mathrm{mg}$ ) and/or CZP before the administration of PTZ to assess their individual and combined effects on clonic seizures.

\section{PTZ-induced clonic seizures}

Following the administration of PTZ, the animals were observed for $30 \mathrm{~min}$ and scored for seizure activity. Clonic seizures were defined as clonus of the whole body lasting for more than $3 \mathrm{~s}$ with loss of the righting reflex. The seizures were scored as: (1) one or more generalized myoclonic twitches of the whole body; (2) repeated clonic seizures of the limbs without loss of righting reflexes; (3) generalized clonic seizures lasting more than $3 \mathrm{~s}$ with loss of righting reflexes, where the animal falls onto one side during the generalized clonus; (4) loss of righting reflexes followed by tonic forelimb seizure; and (5) loss of righting reflexes with tonic seizures in both limbs [33].

\section{Grip strength assessment}

The effects of nobiletin $(50 \mathrm{mg} / \mathrm{kg}$ ) administered alone or in combination with CZP on skeletal muscle strength were assessed using the grip strength test as described by Meyer et al. [34] with minor modifications. The test was conducted 15-20 min after CZP administration. The apparatus consisted of a wire grid $(8 \times 8$ $\mathrm{cm}$ ) connected to an isometric force transducer. Mice were lifted by the tail so they could grasp the grid with their forepaws, then they were gently pulled backwards by the tail until they lost their grip on the grid and were released. The maximum grip force exerted by each mouse before losing its grip was recorded. Muscle strength was expressed in newtons (N).

\section{Chimney test}

We used the chimney test to assess motor performance following the administration of PTZ [35]. The animals were forced to climb backwards up a 30-cm-long plastic tube with a 3-cm inner diameter. Motor impairment was defined as the inability to climb backwards within $60 \mathrm{~s}$

Measurement of neuronal apoptosis We assessed the effect of nobiletin $(50 \mathrm{mg} / \mathrm{kg}$ ) and CZP on neuroapoptosis. Mice were killed by decapitation $36 \mathrm{~h}$ following PTZ administration, and the whole brain was removed from the skull. Neuroapoptosis was assessed using the TUNEL (Terminal deoxynucleotidyl transferase
(TdT) dUTP Nick-End Labeling) assay [36]. Brain tissue sections (5- $\mu \mathrm{m}$ thick) taken from the same level of the hippocampus in each mouse were subjected to analysis using the DeadEnd $^{\mathrm{TM}}$ Fluorometric TUNEL System kit (Promega, Madison, WI, USA). The sections were protected against exposure to direct light during the assay. TUNEL-positive cell counts in the hippocampal regions (CA1 and CA3) were viewed and assessed using NIS-Elements BR imaging processing and analysis software (Nikon Corporation, Tokyo, Japan).

\section{Assessment of brain GABA}

and glutamate levels after PTZ administration

The mice were sacrificed following the administration of PTZ. Brain tissue was immediately isolated, weighed, and homogenized (0.1 M PBS (phosphate-buffered saline)) and GABA and glutamate (Glu) content in the tissues was measured using highperformance liquid chromatography with reference standards [37].

\section{Real-time polymerase chain reaction (RT-PCR)}

RT-PCR analysis was performed to assess the effect of nobiletin on BDNF and TrkB mRNA expression. Total RNA was extracted using TRIzol $^{\circledR}$ reagent (Invitrogen, Carlsbad, CA, USA). The CDNA first strand was synthesized using the RevertAid ${ }^{\text {TM }}$ First Strand CDNA Synthesis Kit (Fermentas, Hanover, MD, USA). PCR was performed using the 7300 Real-Time PCR System (Applied Biosystems, Foster City, CA, USA) with SYBR ${ }^{\circledast}$ green fluorescence. The following primers were used: BDNF forward, 5'-CGAAGAGCTGCTGGATGAG-3'; BDNF reverse, 5'-ATGGGATTACACTTGGTCTCG-3'; TrkBforward, 5'-CCTCCACGGATGTTGCTGA-3'; and TrkB reverse, 5'-GGCTGTTGGTGATACCGAAGTA-3'. GADPH forward, 5'-CCGTATCGGACGCCTGGTTA-3', GADPH reverse, 5'-GGCTGTTGGTGATACCGAAGT A-3'. GAPDH (Glyceraldehyde 3-phosphate dehydrogenase) mRNA was used as an internal control. The PCR products were then separated on agarose gel and visualized following ethidium bromide $(0.05 \%)$ staining. The band 
intensities of the products were analyzed using a Bio-Gel imagery apparatus (Bio-Rad, Hercules, (A, USA).

\section{Western blot analysis of expression levels}

Western blot analysis was performed to measure protein expression levels. Hippocampal tissue homogenized in lysis buffer was incubated for $30 \mathrm{~min}$ on ice. The total protein content of the tissue was determined using a bicinchoninic acid (BCA) assay kit (Bio-Rad). Equal amounts of protein $(60 \mu \mathrm{g})$ from each group were separated using sodium dodecyl sulfate polyacrylamide gel electrophoresis (SDS-PAGE; 10\%), and the proteins were blotted onto polyvinylidene difluoride (PVDF) membranes (Invitrogen). The membranes were blocked in 3\% BSA-TBST (room temperature; $40 \mathrm{~min}$ ) and incubated with specific primary antibodies ( $4^{\circ} \mathrm{C}$ overnight). After washing with TBST, the membranes were incubated for $40 \mathrm{~min}$ at room temperature with a horseradish peroxidase-labeled secondary antibody (1:2,000). The immunoreactive bands were detected and analyzed using an enhanced chemiluminescence method (GE Healthcare, Piscataway, NJ, USA).

\section{Statistical analysis}

Statistical tests were conducted using the Statistical Package for the Social Sciences (SPSS) version 22.0 (SPSS Inc., Chicago, IL, USA). The data were subjected to multiple group comparisons using a one-way analysis of variance (ANOVA) followed by Duncan's multiple range test. P-values $<0.05$ were deemed to indicate statistical significance.

\section{Results}

\section{Nobiletin and CZP reduced PTZ- induced seizure scores}

Systemic administration of nobiletin exerted anticonvulsant effects at doses of $12.5,25$, and $50 \mathrm{mg}$ (Figure 1); however, the effect elicited by the 50-mg dose was more marked than that of the lower doses. Although CZP $(0.02 \mathrm{mg} / \mathrm{kg})$ significantly reduced PTZ-induced seizures ( $p$ $<0.05$ ), the effect was potentiated when the drug was administered in combination with nobiletin, particularly at the 50-mg dose.
Effects of combined nobiletin and CZP on skeletal muscle strength

PTZ administration significantly decreased skeletal muscle strength compared with that of the control group ( $p<0.05$; Figure 2 ). A single injection of CZP $(0.018 \mathrm{mg} / \mathrm{kg})$ prior to the PTZ-induced seizure enhanced skeletal muscle strength. The 50-mg dose of nobiletin significantly improved muscle strength and was more effective than the 12.5 and 25 mg doses; therefore, the 50-mg dose was used in further experiments. The combination of nobiletin and CZP was more effective in improving skeletal muscle strength than either agent alone $(p<$ 0.05).

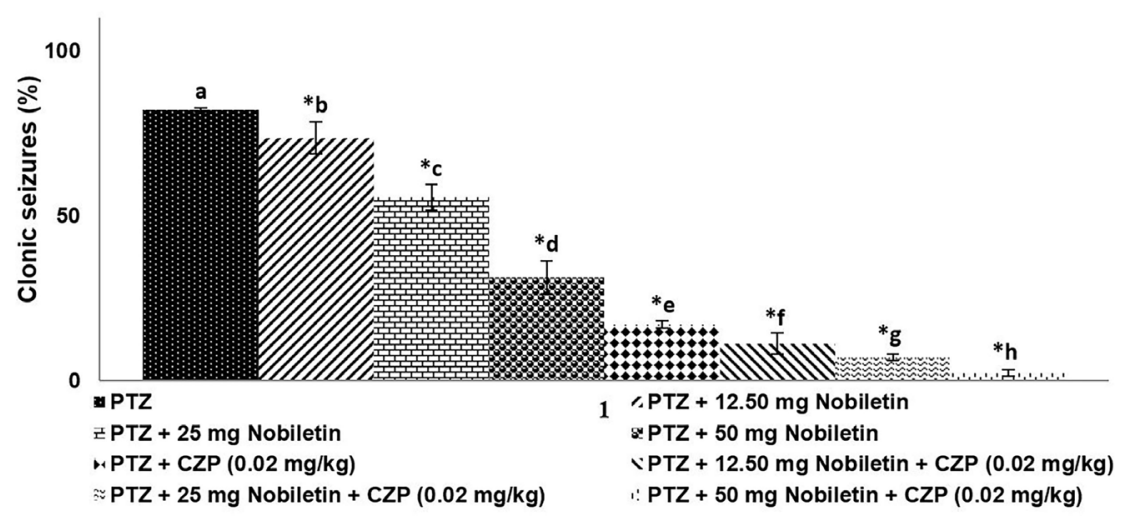

Figure 1. Nobiletin and clonazepam reduced PTZ-induced clonic seizures. Data are expressed as the percentage of animals exhibiting clonic seizures (mean $\pm S D$ ), $n=6 . p<0.05$ as determined by a one-way analysis of variance (ANOVA) followed by Duncan's multiple range test. ${ }^{*} p<0.05$ vs. control. a-h represent mean values from different experimental groups that differ from each other at $\mathrm{p}<0.05$.

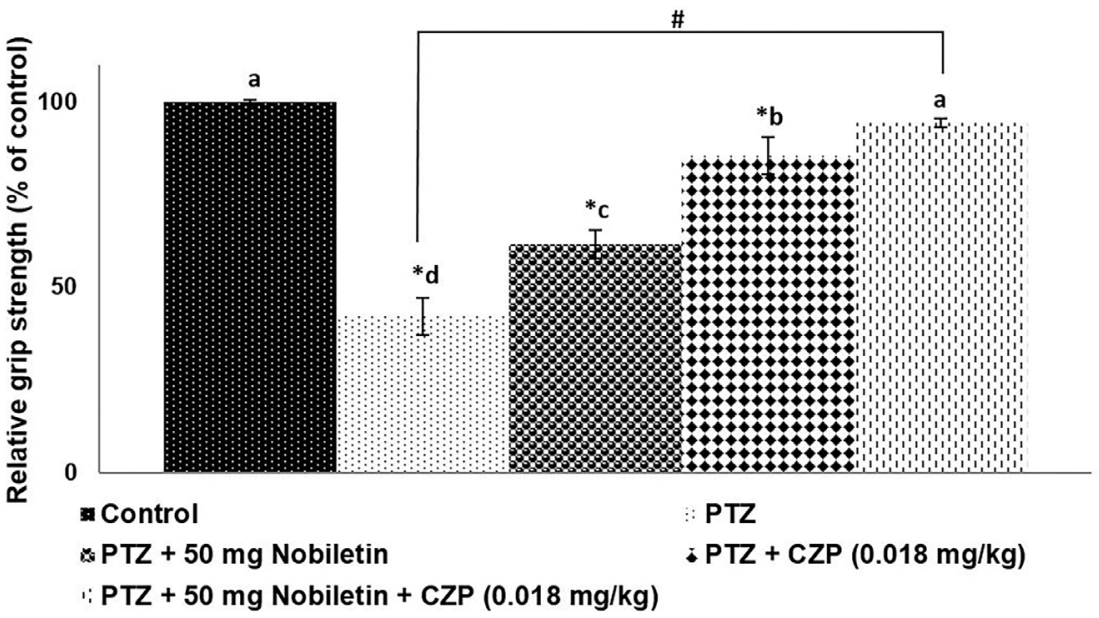

Figure 2. Nobiletin and clonazepam improved skeletal muscle strength after PTZ administration. Data are expressed as mean $\pm S D, n=6$. $p<0.05$ as determined by a one-way ANOVA followed by Duncan's multiple range test. ${ }^{*} p<0.05$ vs. control; \#PTZ vs. PTZ $+50 \mathrm{mg} / \mathrm{kg}$ nobiletin + CZP at $p<0.05$. a-d represent mean values from different experimental groups that differ from each other at $p<0.05$.

\section{Effects of nobiletin and CZP on administration}

PTZ significantly impaired motor coordination: 10 of 12 mice exhibited impaired motor coordination in the chimney test (Figure 3). The performance of mice treated with nobiletin was markedly improved compared with that of PTZ-treated mice. A single injection of CZP $(0.018 \mathrm{mg} / \mathrm{kg})$ improved motor coordination in mice; however, the combined effect of nobiletin and CZP was greater than that of nobiletin or CZP alone (Figure 3). 
Nobiletin significantly reduced neurodegeneration and apoptosis The TUNEL assay revealed a significant increase in TUNEL-positive cell counts in the hippocampus following administration of PTZ $(p<0.05)$. Nobiletin supplementation reduced the number of TUNEL-positive cells, suggesting that the treatment inhibited apoptosis (Figure 4a). TUNEL-positive counts decreased markedly in animals treated with CZP compared with the PTZ group. Moreover, combined CZP and nobiletin treatment significantly reduced the apoptotic cell count $(p<0.05)$.

We measured apoptotic protein expression to better understand the molecular events underlying the anti-apoptotic effect of nobiletin. PTZ produced a marked increase in expression of the pro-apoptotic proteins Bad and Bax (Figure

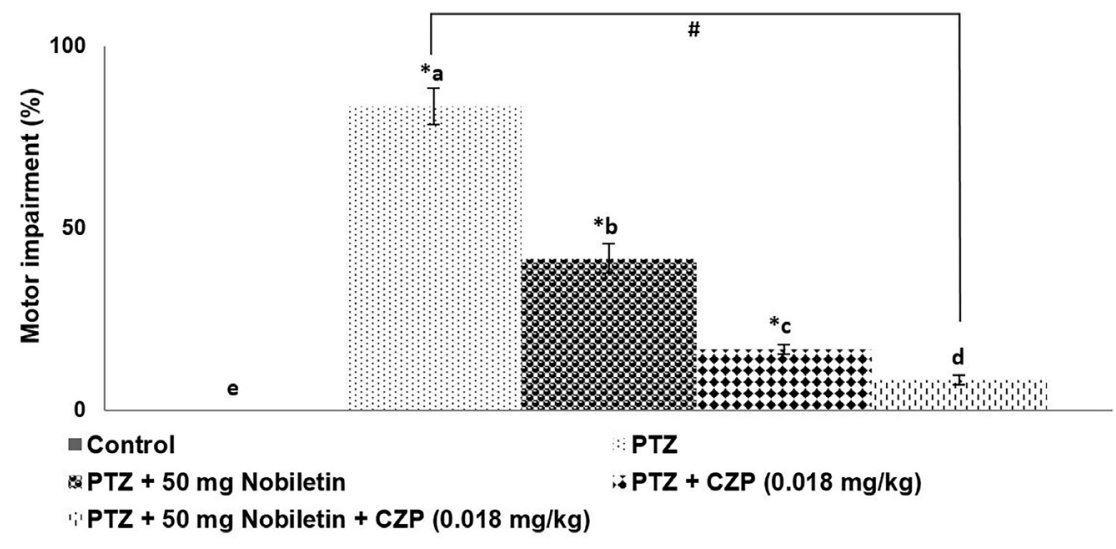

Figure 3. Nobiletin and clonazepam reduced PTZ-induced motor impairment that differ Data are expressed as mean $\pm S D, n=6 . p<0.05$ as determined by a one-way ANOVA followed by Duncan's multiple range test. ${ }^{*} p<$ 0.05 vs. control; \#PTZ vs. PTZ $+50 \mathrm{mg} / \mathrm{kg}$ nobiletin + CZP at $\mathrm{p}<0.05$. a-e represent mean values from different experimental groups from each other at $\mathrm{p}<0.05$.
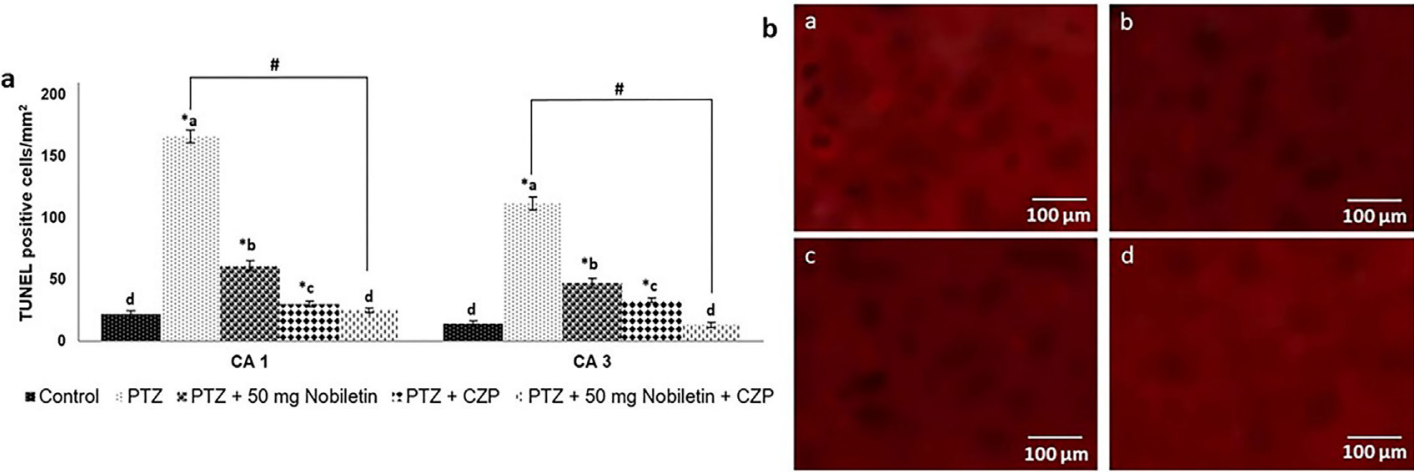

c

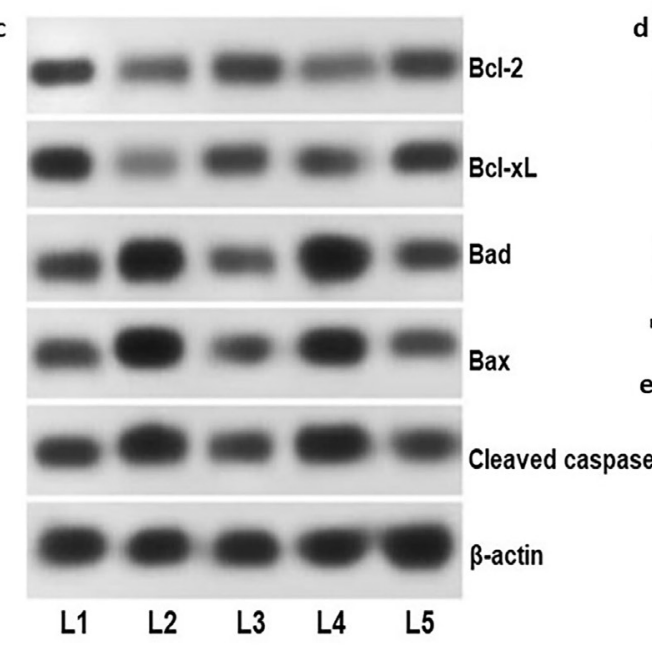

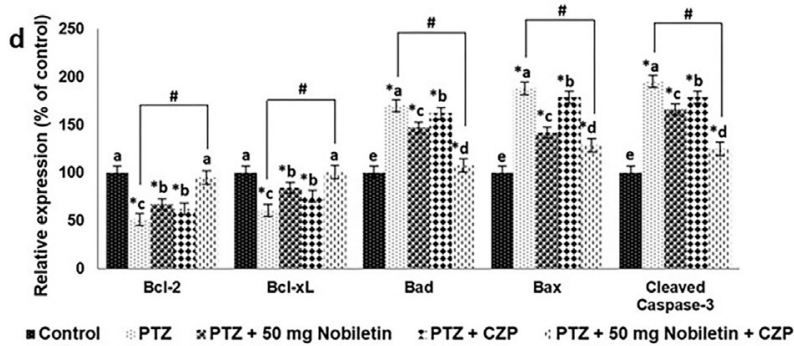

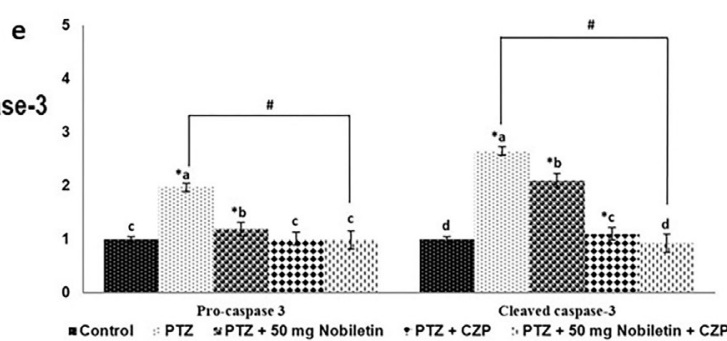

Figure 4. Nobiletin reduced PTZ-induced neuronal apoptosis Nobiletin effectively reduced apoptotic cell counts as determined by TUNEL assay (a and b), Representative Western blot showing modulation of protein expression (c) and Nobiletin modulated the expression of apoptotic pathway proteins ( $d$ and e). Relative expression of proteins with control expression set at 100\%. Data are expressed as mean $\pm S D, n=6 . p<0.05$ as determined by a one-way ANOVA followed by Duncan's multiple range test. * $p<$ 0.05 vs. control; \#PTZ vs. PTZ $+50 \mathrm{mg} / \mathrm{kg}$ nobiletin + CZP at $p<0.05$. a-d represent mean values from different experimental groups that differ from each other at $p<0.05$. L1, control; L2, PTZ; L3, PTZ + 50 mg/kg nobiletin; L4, PTZ + CZP; L5, PTZ + 50 mg/kg nobiletin + CZP. 
$4 b-d)$. Pretreatment with nobiletin reduced the increase in Bad and Bax expression and cleaved caspase-3 levels. $\mathrm{BCl}-2$ and $\mathrm{BCl}-\mathrm{xL}$ expression was significantly upregulated in mice treated with nobiletin $(p<0.05)$, indicating the inhibition of apoptosis. Combined administration of CZP and nobiletin significantly increased $\mathrm{BCl}-2$ and $\mathrm{BCl}-\mathrm{xL}$ expression and reduced the expression of cleaved caspase-3, Bad, and Bax. These findings suggest that nobiletin modulated proteins in the apoptotic pathway to inhibit apoptosis and enhanced the effects of CZP.

\section{Nobiletin regulated BDNF- \\ TrkB signaling following PTZ administration}

The BDNF-TrkB signaling pathway is crucial for epileptogenesis $[38,39]$. BDNF potentiates glutamatergic neurotransmission and inhibits GABAergic transmission [40,41]. The PZTinduced increase in BDNF and TrkB expression at the mRNA and protein levels increased neuronal excitability and subsequently triggered seizures (Figure 5 and 6). Nobiletin pretreatment downregulated the expression of BDNF and TrkB significantly $(p<0.05)$. Although
CZP administration decreased the expression of BDNF and TrkB substantially, the effect was more pronounced with the addition of nobiletin. These findings suggest the anticonvulsant effects of nobiletin and CZP are due in part to inhibition of the BDNF-TrkB signaling pathway.

\section{Effects of nobiletin on GAD65 and GABAA expression}

The expression of GAD65 and $G_{A B A}$ was suppressed in mice injected with PTZ compared with normal controls (Figure 6a, b). Nobiletin upregulated GAD65 and $\mathrm{GABA}_{\mathrm{A}}$ expression significantly ( $p<0.05$ ). CZP administered alone slightly increased the levels of GAD65 and $G_{A B A_{A}}$ expression; however, the effect was enhanced by combined CZP and nobiletin treatment, confirming the antiepileptic potential of nobiletin.

\section{Nobiletin regulation of Glu/GABA levels}

The brain Glu/GABA balance plays a critical role in epileptogenesis $[11,13]$. Nobiletin markedly

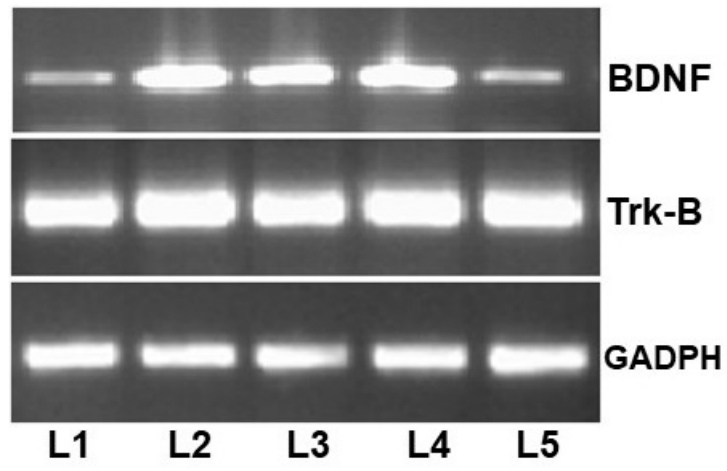

Figure 5. Real-time polymerase chain reaction analysis of mRNA expression L1, control; L2, PTZ; L3, PTZ + 50 mg/ kg nobiletin; L4, PTZ + CZP; L5, PTZ + $50 \mathrm{mg} / \mathrm{kg}$ nobiletin + CZP.
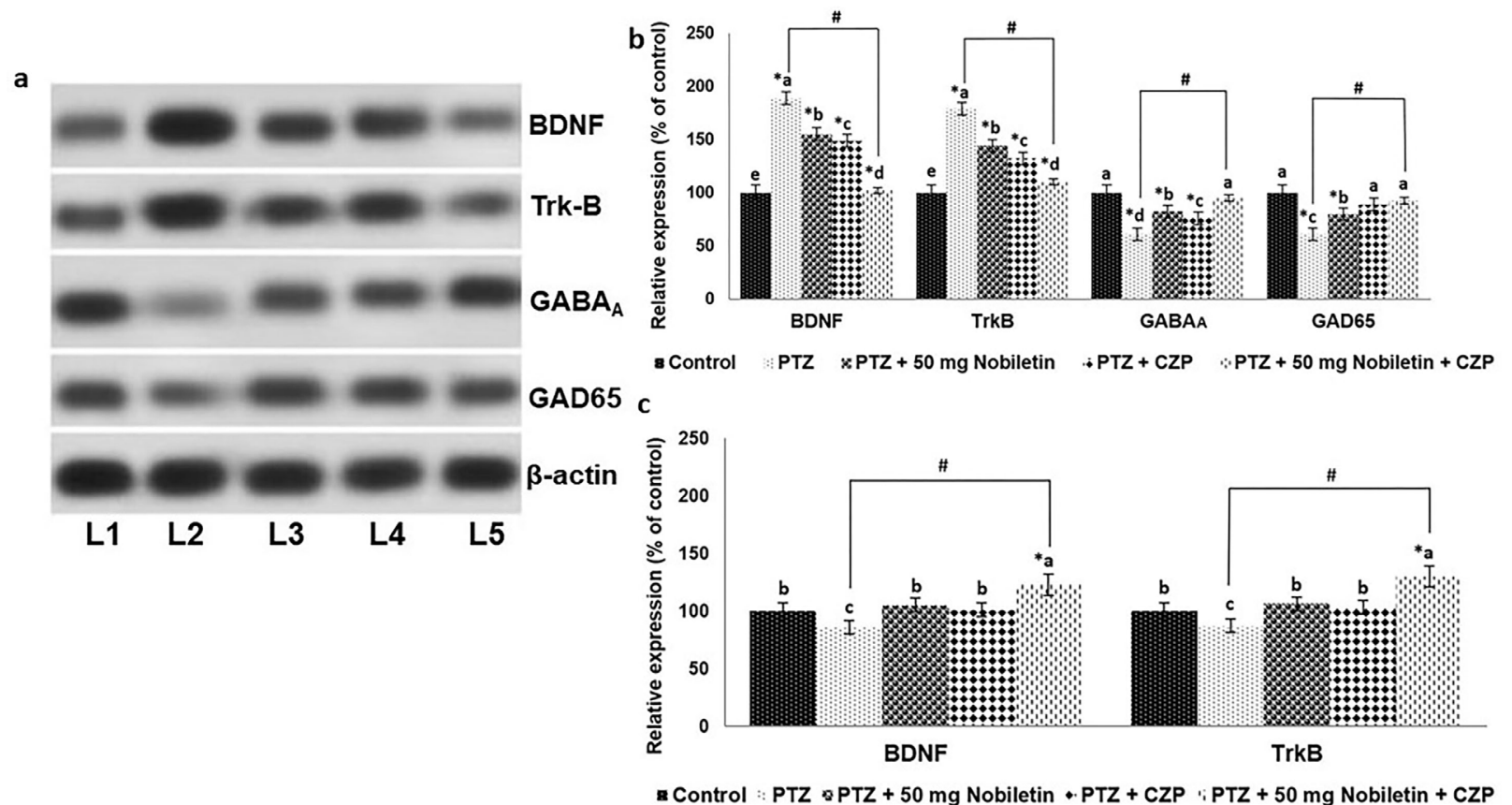

Figure 6. Nobiletin and clonazepam regulate the expression of BDNF-TrkB and GABA and GAD65 proteins A representative Western blot is shown(a) and Relative expression of proteins (b and c) with control expression set at 100\%. L1, control; L2, PTZ; L3, PTZ + $50 \mathrm{mg} / \mathrm{kg}$ nobiletin; L4, PTZ + CZP; L5, PTZ + 50 mg/kg nobiletin + CZP. Data are expressed as mean $\pm S D, n=6 . p<0.05$ as determined by a one-way ANOVA followed by Duncan's multiple range test. * $p<0.05$ vs. control; \#PTZ vs. PTZ + $50 \mathrm{mg} / \mathrm{kg}$ nobiletin + CZP at $\mathrm{p}<0.05$. a-e represent mean values from different experimental groups that differ from each other at $\mathrm{p}<0.05$. 
increased GABA levels and significantly decreased Glu levels ( $p<0.05$ ), thereby normalizing the Glu/GABA ratio (Figure 7). CZP increased GABA levels slightly; however, combined nobiletin and CZP treatment enhanced the effect of CZP alone.

\section{Effect of nobiletin and CZP on PI3K/Akt signaling following PTZ administration}

The PI3K/Akt pathway is expressed widely in developing brain cells and plays a major role in neuronal cell survival [42]. PTZ significantly downregulated the PI3K/Akt pathway (Figure $8 \mathrm{a}-\mathrm{c}$ ) by markedly decreasing expression of the phosphorylated forms of Akt and critical downstream targets, including p-mGSK$3 \beta$, mTORc-1, and mTORc-2. Moreover, PTZ increased the levels of PTEN, a negative regulator of PI3K/Akt signaling. Nobiletin pretreatment activated the PI3K/Akt pathway and increased phosphorylated Akt, GSK-3 $\beta$, mTORc-1, and mTORc-2 levels. Additionally, nobiletin effectively downregulated PTEN, thus promoting cell survival through activation of the PI3K/Akt pathway. CZP treatment resulted in a noticeable increase in the phosphorylation levels of Akt, GSK-3 $\beta$, mTORc-1, and mTORc-2, although the change was not statistically significant. Nobiletin pretreatment enhanced CZP-induced activation of the PI3K/Akt pathway, suggesting that the effects of CZP were potentiated by nobiletin.

\section{Discussion}

The treatment of epilepsy is challenging because of the complexity of the disorder and the serious side effects of currently available medications. Furthermore, onethird of patients are resistant to singledrug therapy [43], highlighting the need for more effective treatments. Concurrent administration of more than one AED has been suggested [3,44]; however, this may increase the incidence of side effects associated with additional drug use. Recent studies have investigated the effectiveness of plant-derived compounds for the treatment of epilepsy alone or in combination with conventional AEDs [45-47]. We assessed the effect of the flavonoid, nobiletin, on the efficacy of CZP.

Nobiletin effectively reduced the severity of PTZ-induced seizures. Moreover, nobiletin pretreatment enhanced the effect of CZP, and the combined treatment was more effective than either agent administered alone. Furthermore, combined treatment with nobiletin and CZP produced a marked increase in muscle strength and motor coordination, as measured by the chimney and grip tests, which was greater than that achieved by CZP or nobiletin alone. These findings highlight the potentiating effect of nobiletin.

Previous studies have shown that seizures induce neuronal death in the brain [48-50]. We found clear evidence of neuronal apoptosis in the CA1 and CA3 hippocampal regions following PTZ administration. Nobiletin exerted a protective effect by significantly reducing the apoptotic cell count measured using the TUNEL assay. The significant elevations in caspase-3 levels and Bax and Bad protein expression confirmed that PTZ increased apoptosis in the mice. Nobiletin upregulated $\mathrm{BCl}-\mathrm{xL}$ and $\mathrm{BCl}-2$ and downregulated the expression of caspase-3, Bad, and Bax, suggesting that the flavonoid suppressed apoptosis. Nobiletin pretreatment significantly enhanced the protective effect of CZP, suggesting that the nobiletin/CZP-mediated modulation of protein expression may reduce neuronal cell loss. These results could be due to the additive effects of nobiletin administered with CZP.

The BDNF-TrkB signaling pathway plays an important role in epilepsy $[15,51]$. Epileptogenic insult induces BDNF synthesis and activates TrkB receptors [15,52]. We observed a marked increase in the expression of BDNF and TrkB after the administration of PTZ, suggesting that the convulsive agent activates the BDNF-TrkB pathway. Nobiletin treatment potentially inhibited BDNF-TrkB activity, thereby downregulating the BDNF signaling pathway. As reported previously, we found that the combination of CZP and nobiletin was more effective than either agent alone. Thus, reflecting the possible synergistic or additive effects of both the drugs. However,

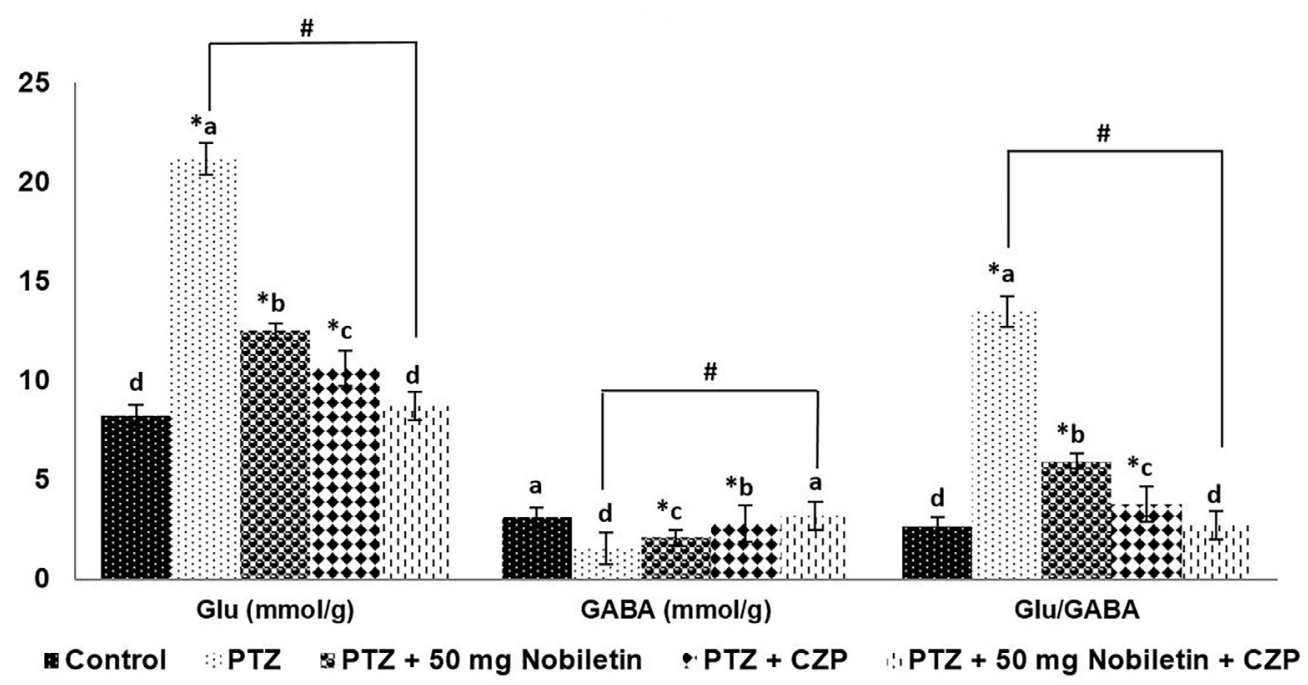

Figure 7. Nobiletin and clonazepam restored the Glu/GABA balance. Data are expressed as mean \pm SD, $\mathrm{n}=6 . p<0.05$ as determined by a one-way ANOVA followed by Duncan's multiple range test. * $\mathrm{p}<0.05$ vs. control; \#PTZ vs. PTZ $+50 \mathrm{mg} / \mathrm{kg}$ nobiletin $+C Z P$ at $p<0.05$. a-d represent mean values from different experimental groups that differ from each other at $\mathrm{p}<0.05$ 


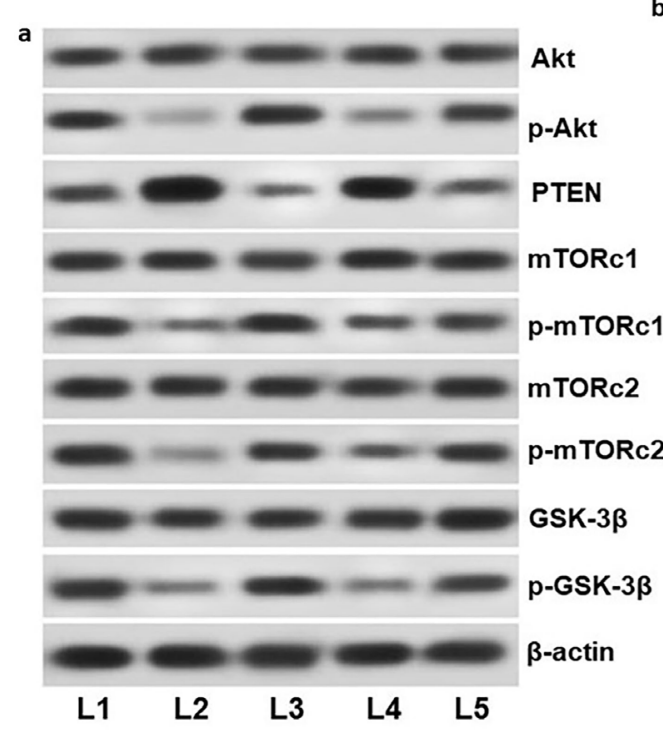

Figure 8. Nobiletin and clonazepam upregulated PI3K/Akt signaling following PTZ-induced seizure. A representative Western blot is shown (a) and Relative expression of proteins ( $\mathrm{b}$ and $\mathrm{c}$ ) with control expression set at 100\%. L1, control; L2, PTZ; L3, PTZ + $50 \mathrm{mg} / \mathrm{kg}$ nobiletin; L4, PTZ + CZP; L5, PTZ + 50 mg/kg nobiletin + CZP. sData are expressed as mean $\pm S D, n=6$. $p<0.05$ as determined by a one-way ANOVA followed by Duncan's multiple range test. ${ }^{*} p<0.05$ vs. control; \#PTZ vs. PTZ + 50 mg/kg nobiletin + CZP at $p<0.05$. a-d represent mean values from different experimental groups that differ from each other at $p<0.05$.

how nobiletin potentiated the effects of CZP is yet to be investigated. Several previous studies have shown that BDNF-TrkB signaling promotes epileptiform activity [38,53]. Thus, downregulation of BDNF-TrkB may contribute to the suppression of seizures.

The balance between excitatory and inhibitory neurotransmitters has a critical effect on epileptogenesis [54]. PTZ disturbs the Glu/GABA balance by increasing Glu and decreasing GABA levels. Combined nobiletin/CZP treatment restored the Glu/ GABA balance by increasing GABA levels and markedly lowering Glu levels. Nobiletin increased the protein expression of GAD65, the rate-limiting enzyme in GABA synthesis $[13,37]$, and $G_{A B A}$. Recent studies have suggested GAD65 and $\mathrm{GABA}_{A^{\prime}}$ the primary GABA receptor, as targets for novel AEDs $[11,13,54]$. The ability to restore the Glu/GABA balance and increase the expression of GAD65 and $G A B A_{A}$ suggests that nobiletin has potent antiepileptic activity. BDNF has been shown to simultaneously potentiate glutamatergic and inhibit GABAergic neurotransmission [15,55]; thus, combined nobiletin/CZP modulation of BDNF expression and BDNF-TrkB signaling may have contributed to the improved Glu/ GABA balance. Although CZP altered Glu/ GABA levels and BDNF-TrkB signaling, the effects were noticeably more pronounced with nobiletin pretreatment as seen in the expressions of apoptotic proteins.

The PI3K/Akt signaling pathway is a key regulatory pathway involved in neuronal cell proliferation, growth, and cell survival [41]. Activation of the pathway has been shown to exert neuroprotective effects against seizures [56,57]. PTZ administration downregulated the pathway as evidenced by a marked decrease in the activation of Akt, a key downstream regulator of the pathway that exerts neuroprotective actions [56]. Phosphorylation of crucial proteins in the pathway, including mTORc1, mTORc2, and GSK-3 $\beta$, was suppressed significantly by PTZ administration. The PI3K/ Akt signaling pathway was activated by nobiletin, as indicated by marked increases in the expression of phosphorylated Akt, mTORc1, mTORc2, and GSK-3 $\beta$ together with the suppression of PTEN levels. Previous studies have found that activated Akt inhibited the expression of the Bad and Bax proteins and induced $\mathrm{BCl}-2$ and $\mathrm{Bcl}-\mathrm{xL}$ [57-59]. Although,
CZP administered singly, activated the PI3K/Akt pathway, the effect of combined CZP/nobiletin treatment was greater than that of CZP alone, indicating the synergistic or additive effect of both the drugs. Therefore, nobiletin/CZPmediated inhibition of apoptosis may result from a direct action of nobiletin on apoptotic pathway proteins, due in part to activation of the PI3K/Akt pathway.

\section{Conclusion}

Our findings demonstrate that nobiletin has antiepileptic properties that protect against PTZ-induced seizures. Moreover, nobiletin potentiated the effects of CZP; CZP in combination with nobiletin significantly reduced the severity of seizures via the modulation of GAD65/GABA $A_{A}$ expression and the BDNF-TrkB and PI3K/Akt signaling pathways. Our findings suggest that nobiletin is a potent antiepileptic candidate drug that could be administered alone or in combination with conventional AEDs. However, clinical studies are needed to further investigate the possible molecular events underlying the antiepileptic effects of nobiletin. 
[1] Duncan JS, Sander JW, Sisodiya SM, Walker MC. Adult epilepsy. Lancet 2006;367:1087-100.

[2] Perucca E, French J, Bialer M. Development of new antiepileptic drugs: challenges, incentives, and recent advances. Lancet Neurol. 2007;6:793-804.

[3] Kwan P, Brodie MJ. Early identification of refractory epilepsy. N Engl J Med. 2000a;342: 314-19.

[4] Luszczki JJ, Antkiewicz-Michaluk L, Czuczwar SJ. Isobolographic analysis of interactions between 1-methyl-1,2,3,4tetrahydroisoquinoline and four conventional antiepileptic drugs in the mouse maximal electroshock-induced seizure model. Eur J Pharmacol. 2009a;602:298-305.

[5] Schmitz B. Effects of antiepileptic drugs on mood and behavior. Epilepsia 2006;47:28-33.

[6] Luszczki JJ, Wojcik-Cwikla J, Andres MM, Czuczwar SJ. Pharmacological and behavioural characteristics of interactions between vigabatrin and conventional antiepileptic drugs in pentylenetetrazole-induced seizures in mice: an isobolographic analysis. Neuropsychopharmacology 2005;30:958-73.

[7] Kumar A, Lalitha S, Mishra J. Possible nitric oxide mechanism in the protective effect of hesperidin against pentylenetetrazole (PTZ)induced kindling and associated cognitive dysfunction in mice. Epilepsy Behav. 2013;29:103-111.

[8] Suzdak PD, Jansen JA. A review of the preclinical pharmacology of tiagabine: a potent and selective anticonvulsant GABA uptake inhibitor. Epilepsia 1995;36:612-26.

[9] Vianello M, Tavolato B, Giometto B. Glutamic acid decarboxylase autoantibodies and neurological disorders. Neurol Sci. 2002;23:145-51.

[10] Ha JH, Lee DU, Lee JT, Kim JS, Yong CS, Kim JA, et al. 4-Hydroxybenzaldehyde from Gastrodia elata B1. is active in the antioxidation and GABAergic neuromodulation of the rat brain. J Ethnopharmacol. 2000;73:329-33.

[11] Awad R, Ahmed F, Bourbonnais-Spear N, Mullally M, Ta CA, Tang A, et al. Ethnopharmacology of Q'eqchi' maya antiepileptic and anxiolytic plants: effects on the GABAergic system. J Ethnopharmacol. 2009;125:257-64.

[12] Yang DB, Wang L, Huang M, Yu JM, Wang XM, Luo JM. Effects of pre-treatment with repetitive transcranial magnetic stimulation on development of seizures induced by pilocarpine and expression of GAD65 in rat hippocampus. Chin J Clin Neurosci. 2009;17:337-40.

[13] Xiang J, Jiang Y. Antiepileptic potential of matrine via regulation the levels of gamma-aminobutyric acid and glutamic acid in the brain. Int J Mol Sci. 2013;14:23751-761.

[14] Kafitz KW, Rose CR, Thoenen H, Konnerth A. Neurotrophin evoked rapid excitation through TrkB receptors. Nature 1999;401:918-21.

[15] Binder DK, Croll SD, Gall CM, Scharfman HE. BDNF and epilepsy: too much of a good thing? Trends Neurosci. 2001;24:47-53.

[16] Brunet A, Datta SR, Greenberg ME. Transcription-dependent and independent control of neuronal survival by the PI3K-Akt signaling pathway. Curr Opin Neurobiol. 2001;11:297-305.
[17] Jaworski J, Sheng M. The growing role of mTOR in neuronal development and plasticity. Mol Neurobiol. 2006;34:205-19.

[18] Swiech L, Perycz M, Malik A, Jaworski J. Role of mTOR in physiology and pathology of the nervous system. Biochim Biophys Acta. 2008;1784:116-32.

[19] Mantoan L, Walker M. Treatment options in juvenile myoclonic epilepsy. Curr Treat Options Neurol. 2011;13:355-70.

[20] Perna G, Alciati A, Riva A, Micieli W, Caldirola D. Long-term pharmacological treatments of anxiety disorders: an updated systematic review. Curr Psychiatry Rep. 2016;18:23.

[21] Czapinski P, Blaszczyk B, Czuczwar SJ. Mechanisms of action of antiepileptic drugs. Curr Top Med Chem. 2005;5:3-14.

[22] Xie T, Wang WP, Mao ZF, Qu ZZ, Luan SQ, Jia LJ, et al. Effects of epigallocatechin-3-gallate on pentylenetetrazole-induced kindling, cognitive impairment and oxidative stress in rats. Neurosci Lett. 2012;516:237-41.

[23] Rahmati B, Khalili M, Roghani M, Ahghari P. Anti-epileptogenic and antioxidant effect of Lavandula officinalis aerial part extract against pentylenetetrazol-induced kindling in male mice. J Ethnopharmacol. 2013;148:152-57.

[24] Chen J, Chen AY, Huang H, Ye X, Rollyson WD, Perry HE, et al. The flavonoid nobiletin inhibits tumor growth and angiogenesis of ovarian cancers via the Akt pathway. Int J Oncol. 2015;46:2629-38.

[25] Cheng HL, Hsieh MJ, Yang JS, Lin CW, Lue KH, Lu KH, et al. Nobiletin inhibits human osteosarcoma cells metastasis by blocking ERK and JNK-mediated MMPs expression. Oncotarget 2016;7:35208-23.

[26] Guo S, Qiu P, Xu G, Wu X, Dong P, Yang G, et al. Synergistic anti-inflammatory effects of nobiletin and sulforaphane in lipopolysaccharide-stimulated RAW 264.7 cells. J Agric Food Chem. 2012;60:2157-64.

[27] Nakajima A, Ohizumi Y, Yamada K. Anti-dementia activity of nobiletin, a citrus flavonoid: A review of animal studies. Clin Psychopharmacol Neurosci. 2014;12:75-82.

[28] Cho HW, Jung SY, Lee GH, Cho JH, Choi IY. Neuroprotective effect of Citrus unshiu immature peel and nobiletin inhibiting hydrogen peroxide-induced oxidative stress in HT22 murine hippocampal neuronal cells. Pharmacognosy Magazine 2015;11:S284-89.

[29] Garber JC. Committee for the update of the guide for the care and use of laboratory animals. In: Guide for the care and use of laboratory animals. $8^{\text {th }}$ ed. National Academy of Sciences, USA; 2011.

[30] Luszczki JJ, Czuczwar P, Cioczek-Czuczwar A, Dudra-Jastrzebska $M$, Andres-Mach $M$, Czuczwar SJ. Effect of arachidonyl-2'chloroethylamide, a selective cannabinoid CB1 receptor agonist, on the protective action of the various antiepileptic drugs in the mouse maximal electroshock-induced seizure model. Prog Neuropsychopharmacol Biol Psychiatry 2010;34:18-25.

[31] Luszczki JJ, Andres-Mach M, Barcicka-Klosowska B, FlorekLuszczki M, Haratym-Maj A, Czuczwar SJ. Effects of WIN $55,212-2$ mesylate (a synthetic cannabinoid) on the protective action of clonazepam, ethosuximide, phenobarbital and valproate 
against pentylenetetrazole- induced clonic seizures in mice. Prog Neuropsychopharmacol Biol Psychiatry 2011;35:1870-76.

[32] Dudra-Jastrzebska M, Andres-Mach MM, Ratnaraj N, Patsalos PN, Czuczwar SJ, Luszczki JJ. Isobolographic characterization of the anticonvulsant interaction profiles of levetiracetam in combination with clonazepam, ethosuximide, phenobarbital and valproate in the mouse pentylenetetrazole-induced seizure model. Seizure 2009;18:607-14.

[33] Loscher W, Honack D, Fassbender CP, Nolting B. The role of technical, biological and pharmacological factors in the laboratory evaluation of anticonvulsant drugs. III. Pentylenetetrazole seizure models. Epilepsy Res. 1991;8:171-89.

[34] Meyer OA, Tilson HA, Byrd WC, Riley MT. A method for the routine assessment of fore and hindlimb grip strength of rats and mice. Neurobehav Toxicol. 1979;1:233-36.

[35] Boissier JR, Tardy J, Diverres JC. Une nouvelle methode simple pour explorer l'action tranquilisante: le test de la cheminee. Med Exp (Basel) 1960;3:81-84.

[36] Li Y, Liang G, Wang S, Meng Q, Wang Q, Wei H. Effects of fetal exposure to isoflurane on postnatal memory and learning in rats. Neuropharmacology 2007;53:942-50.

[37] Yang T, Kong B, Gu JW, Kuang YQ, Cheng L, Yang WT, et al. Anticonvulsant and sedative effects of paederosidic acid isolated from Paederia scandens (Lour.) Merrill. in mice and rats. Pharmacol Biochem Behav. 2013; 111:97-101.

[38] Lahteinen S, Pitkanen A, Saarelainen T, Nissinen J, Koponen E, Castrén E. Decreased BDNF signaling in transgenic mice reduces epileptogenesis. Eur J Neurosci. 2002;15:721-34.

[39] Wang Y, Qi JS, Kong S, Sun Y, Fan J, Jiang M, et al. BDNF-TrkB signaling pathway mediates the induction of epileptiform activity induced by a convulsant drug cyclothiazide. Neuropharmacology 2009;57:49-59.

[40] Kang H, Schuman EM. Long-lasting neurotrophin induced enhancement of synaptic transmission in the adult hippocampus. Science 1995;267:1658-62.

[41] Zhu WJ, Roper SN. Brain-derived neurotrophic factor enhances fast excitatory synaptic transmission in human epileptic dentate gyrus. Ann Neurol. 2001;50:188-94.

[42] Duman RS, Voleti B. Signaling pathways underlying the pathophysiology and treatment of depression: novel mechanisms for rapid-acting agents. Trends Neurosci. 2012;35:47-56.

[43] Deckers CL, Czuczwar SJ, HeksterYA, Keyser A, Kubova H, Meinardi H, et al. Selection of antiepileptic drug polytherapy based on mechanisms of action: the evidence reviewed. Epilepsia 2000;41:1364-74.

[44] Kwan P, Brodie MJ. Epilepsy after the first drug fails: substitution or add-on? Seizure 2000b;9:464-68

[45] Johannessen-Landmark C, Patsalos PN. Interactions between antiepileptic drugs and herbal medicines. Bol Latinoam Caribe Plant Med Aromaticas 2008;7:108-118.

[46] Luszczki JJ, Czarnecki R, Wojtal K, Borowicz KK, Czuczwar SJ. Agmatine enhances the anticonvulsant action of phenobarbital and valproate in the mouse maximal electroshock seizure model. J Neural Transm. 2008;115:1485-93.

[47] Luszczki JJ, Wojda E, Andres-Mach M, Cisowski W, Glensk M, Glowniak K, et al. Anticonvulsant and acute neurotoxic effects of imperatorin, osthole and valproate in the maximal electroshock seizure and chimney tests in mice: a comparative study. Epilepsy Res. 2009b;85:293-99.

[48] Diemer NH, Jorgensen MB, Johansen FF, Sheardown M, Honore T. Protection against ischemic hippocampal CA1 damage in the rat with a new non-NMDA antagonist NBQX. Acta Neurol Scand. 1992;86:4549.

[49] Gee CE, Benquet P, Raineteau O, Rietschin L, Kirbach SW, Gerber $U$. NMDA receptors and the differential ischemic vulnerability of hippocampal neurons. Eur J Neurosci. 2006;23:2595-603.

[50] Dos-Anjos S, Martinez-Villayandre B, Montori S, Regueiro-Purrinos MM, Gonzalo-Orden JM, Fernandez-Lopez A. Transient global ischemia in rat brain promotes different NMDA receptor regulation depending on the brain structure studied. Neurochem Int. 2009;54:180-85

[51] Liu X, Liu J, Liu J, Liu XL, Jin LY, Fan W, et al. BDNF-TrkB signaling pathway is involved in pentylenetetrazole-evoked progression of epileptiform activity in hippocampal neurons in anesthetized rats. Neurosci Bull. 2013;29:565-75.

[52] Reibel S, Depaulis A, Larmet Y. BDNF and epilepsy-the bad could turn out to be good. Trends Neurosci. 2001;24:318-19.

[53] Papaleo F, Silverman JL, Aney J, Tian Q, Barkan CL, Chadman KK, et al. Working memory deficits, increased anxiety-like traits, and seizure susceptibility in BDNF overexpressing mice. Learn Mem. 2011;18:53444

[54] Svenningsen AB, Madsen KD, Liljefors T, Stafford Gl, Stafford Gl, van Staden J, et al. Biflavones from Rhus species with affinity for the GABAA/benzodiazepine receptor. J Ethnopharmacol. 2006;103:27680.

[55] Tanaka T, Saito $H$, Matsuki N. Inhibition of GABAA synaptic responses by brain-derived neurotrophic factor (BDNF) in rat hippocampus. J Neurosci. 1997;17:2959-66.

[56] Henshall DC, Araki T, Schindler CK, Lan JQ, Tiekoter KL, Taki W, et al. Activation of $\mathrm{Bcl}$-2-associated death protein and counter-response of Akt within cell populations during seizure induced neuronal death. J Neurosci. 2002;22:8458-65.

[57] Xue Y, Xie N, Cao L, Zhao X, Jiang H, Chi Z. Diazoxide preconditioning against seizure-induced oxidative injury is via the PI3K/Akt pathway in epileptic rat. Neurosci Lett. 2011;495:130-34.

[58] Asnaghi L, Calastretti A, Bevilacqua A, D’Agnano I, Gatti G, Canti G, et al. $\mathrm{BCl}-2$ phosphorylation and apoptosis activated by damaged microtubules require mTOR and are regulated by Akt. Oncogene 2004;23:5781-91.

[59] Atif F, Yousuf S, Stein DG. Anti-tumor effects of progesterone in human glioblastoma multiforme: role of PI3K/Akt/mTOR signaling. J Steroid Biochem Mol Biol. 2015;146:62-73. 